\title{
Timing Recovery with SAW Transversal Filters in the Regenerators of Undersea Long-Haul Fiber Transmission Systems
}

\author{
ROBERT L. ROSENBERG, SENIOR MEMBER, IEEE, CHRISTODOULOS CHAMZAS, MEMBER, IEEE, \\ AND DANIEL A. FISHMAN
}

\begin{abstract}
Surface-acoustic-wave (SAW) filters have been generally accepted as the best available choice for timing recovery in repeatered transoceanic fiber transmission systems operating in the range of $\mathbf{3 0 0}$ Mbit/ $\mathrm{s}$ as well as in other digital transmission systems operating from approximately 0.1 to 1 or $2 \mathrm{Gbit} / \mathrm{s}$. The SAW technology is ready and reliable, filter size is consistent with the severe space limitations of undersea repeater housings, and cost is reasonable, especially for the transversal (tapped-delay-line) filter type. This paper updates the relationship between system performance and the characteristics of transversal SAW filters, which have a finite impulse response and non-minimum-phase behavior. New results relate to passband ripple (typical in these filters), thermal characteristics, and the impact of the delay-line structure on filter "ringing" time.
\end{abstract}

\section{INTRODUCTION}

A $\mathrm{S}$ with other aspects of transoceanic fiber transmission systems, ongoing development has fostered a growing sophistication in the regenerator timing-recovery loop. A broad-brush study of repeatered systems with surface-acoustic-wave (SAW) retiming filters, reported about two years ago [1], has already become dated. Progress since that time on three important topics is summarized here. Two of the topics came to the fore when a firm decision was made in favor of transversal SAW filters for the transatlantic cable. Such filters are notorious for the difficulty of eliminating passband ripple. The effects of ripple on timing jitter and alignment jitter have been characterized analytically [2], with results that are somewhat alarming under worst-case conditions. Fortunately, the worst-case conditions are substantially softened [3] by natural variations in the passband shapes of manufactured filters. The jitter situation is described in Section III. Another notable consequence of using the transversal type of filter is that its effective "ringing" time is much longer than the ringing time of a minimum-phase (resonator) filter with the same $3-\mathrm{dB}$ fractional bandwidth (" $1 / Q$ "). The reasons and the system implications are discussed in Section IV.

Finally, the moderate temperature variability of SAW filter characteristics on quartz has proved to be more useful than the absolute temperature stability that has been pursued for many years. The temperature variation of filter

Manuscript received July 1, 1984; revised August 1, 1984. The authors are with AT\&T Bell Laboratories, Holmdel, NJ 07733. phase can be employed to compensate much of the phasetemperature variation of the timing-loop electronics [4]. As a result, the thermal offset of the decision point from its optimum location in the eye diagram can be well controlled over the required temperature range. The compensation scheme is described in Section $V$ where we also assess the cost, an increase in static frequency detuning. Results are summarized in Section VI.

To establish terminology and simplify later discussion, we begin with a basic picture of a SAW transversal filter in Section II.

\section{A Basic SAW Transversal Filter}

The simplest form of the SAW transversal filter is shown in Fig. 1(a) [5]. Piezoelectric transducers, which convert an RF electrode voltage difference into propagating SAW's or vice versa, are arranged as transmitting and receiving endfire arrays along the propagation axis. Each transducer is a pair of interdigitated metallic-film combs deposited on a piezoelectric substrate (quartz in the case of narrow-band timing-recovery filters). A voltage applied at the bus bars of the transmitter combs creates strong fields of alternating sign between successive pairs of fingers to generate localized alternating strains (acoustic standing waves equivalent to a pair of running waves) in the substrate. The inverse process occurs at the receiver.

Contrary to the case of minimum-phase (resonator) filters, the amplitude and phase characteristics of a transversal filter are nearly independent of each other. The transmission phase of a narrow-band transversal filter is linear in frequency to a good approximation since the phase is determined primarily by constant-velocity acoustic propagation over a path of many wavelengths between transmitter and receiver.

In scattering terms, the simple transducers shown are 3-ports [6], [7], with an electrical port at the bus bars and two acoustic ports at the ends of each array. As with any narrow-band filter, the passband shape is sensitive to the terminations at the electrical ports. It is characteristic of these 3-ports that if the impedance match at the electrical port is improved to raise the transduction efficiency (thereby reducing the insertion loss of the filter), there is an 


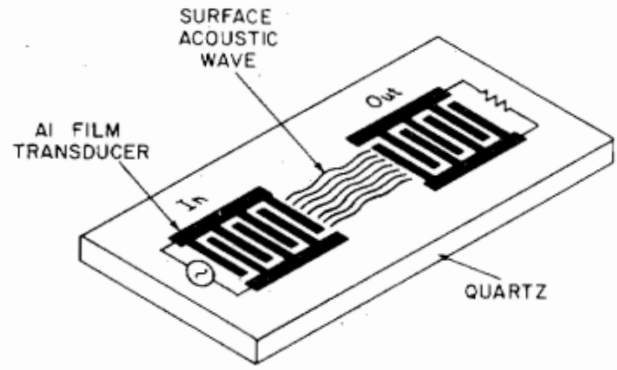

(a)

(1) FIRST TRANSIT

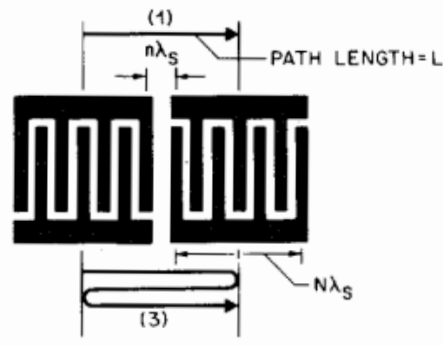

(3) TRIPLE TRANSIT

(b)

Fig. 1. (a) Basic structure of a transversal filter. The electrical source and load are shown schematically to indicate standard connections to the bus-bar pairs of the transducers. (b) Illustration of triple-transit reflections, which interfere coherently with simultaneously received first transits. For narrow-band retiming filters, the number of periods $N_{T}$ per transducer is often several hundred. $\lambda_{s}$ is the "synchronous frequency" at which the acoustic wavelength matches the transducer period. A quarter-wave change in the transducer separation $n \lambda_{s}$ can convert a dip to a peak at passband center [2]. Comparable phase changes over the propagation path length $L$ may occur through random variations of $\lambda_{s}$ caused by fabrication errors.

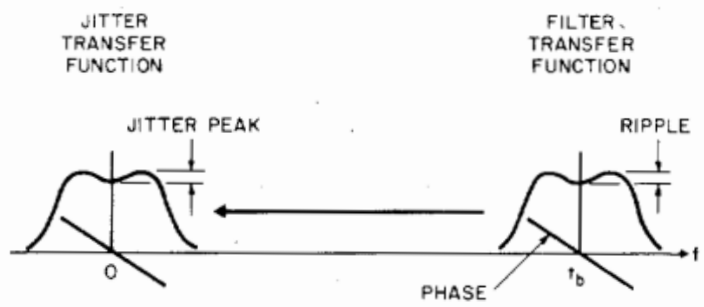

Fig. 2. Illustration of the simple relationship between a ripply filter transfer function and the associated jitter transfer function when the filter transfer function has symmetric amplitude and antisymmetric phase about the baud frequency. In that case, the ripple translates into a jitter peak.

attendant increase in the reflection of incoming acoustic waves at the acoustic ports [6], [7]. In a filter, the result is multiple acoustic reflections between transducers. The largest of these is the "triple-transit" reflection shown in Fig. 1(b). The triple transit is a delayed version of the directly transmitted signal and interferes with it coherently to produce a passband ripple (Fig. 2) that is difficult to suppress. Although there is a voluminous literature dealing with ripple reduction, in hardware the ripple never goes away entirely. The jitter studies were undertaken to determine the consequences of ripple effects in a long chain of regenerators and to learn how much ripple might be acceptable in a transoceanic system.
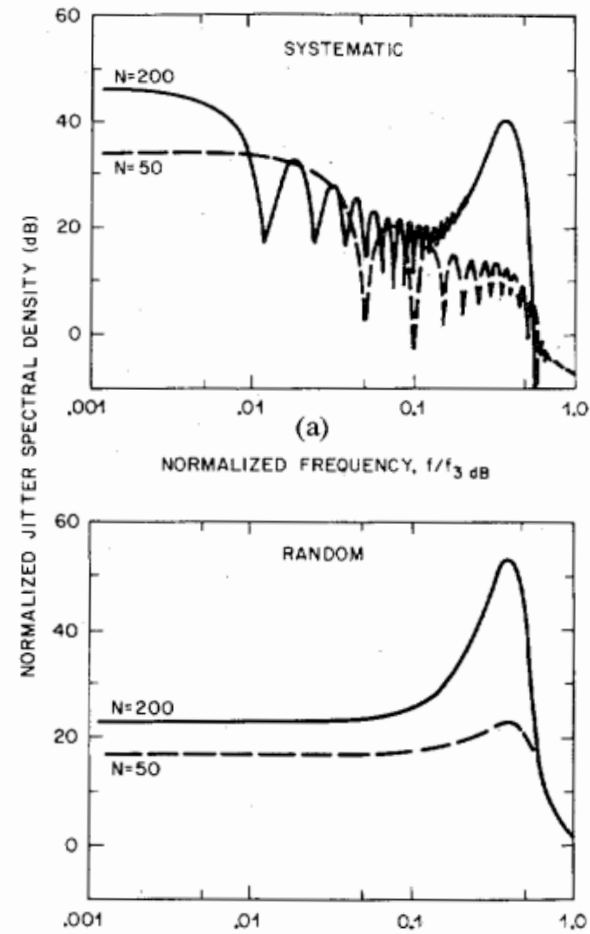

(b)

Fig. 3. Spectra of (a) systematic and (b) random jitter accumulated through 50 and 200 cascaded regenerators with identical jitter peaks of $0.2 \mathrm{~dB}$ and $Q$ 's of 800 . The vertical scale has been normalized to $0 \mathrm{~dB}$ at low frequencies for the jitter spectral density after one regenerator. The horizontal scale is normalized to the $3-\mathrm{dB}$ point of the ripple-free model [2].

\section{PASSBAND RipPle EFFECTS ON JitTER}

The first question that arises is whether ripple on the filter transmission passband can cause jitter peaking in the associated regenerator transfer function for phase jitter [1], [8]. The answer turns out to be simple in the case of a filter with symmetric ripple and linear phase. The phase-jitter transfer function is then effectively the same as the filter transfer function [9] translated to baseband and normalized to 1 at dc, as shown in Fig. 2. The ripple becomes the jitter peak. Once the presence of a jitter peak is established, there is a real risk that timing jitter accumulation along a chain of regenerators can run away exponentially with the number of regenerators [8]. The remaining question is "Where along the chain does runaway growth take over?" The first studies [2] examined this question by applying the Chapman model [9] of cascaded regenerators to compute timing jitter accumulation and alignment jitter when all filters have the same symmetric ripple magnitude and peak frequencies. The filter model used for calculation was based on the crossed-field circuit model [2], [6] of transducers like those in Fig. 1, and included triple transits to describe the ripple in a natural way. The transmitting and receiving transducers were assumed to be identical. Sample results of the computations are shown in Figs. 3-5.

Fig. 3 shows computed spectra of both systematic and random timing jitter for chains of 50 and 200 regenerators. All the filters in the chain are assumed to be identical, with a ripple of $0.2 \mathrm{~dB}$ and a $Q$ of about 800 . The vertical scale 


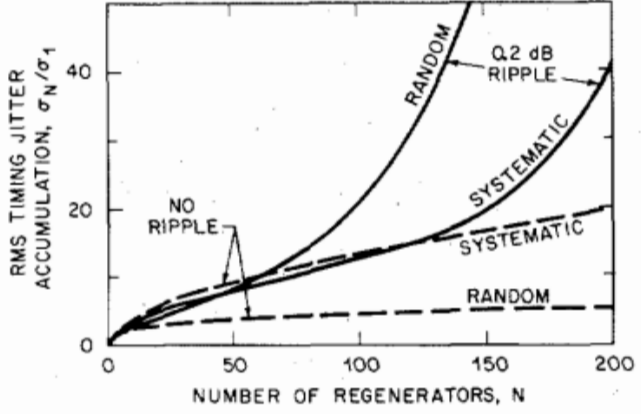

Fig. 4. rms timing jitter accumulation as a function of $N$, the number of identical cascaded regenerators in a chain. Both the systematic and random jitter components are shown for a peak of $0.2 \mathrm{~dB}$ in the jitter transfer function. Curves are also shown. for the case of no jitter peaking.

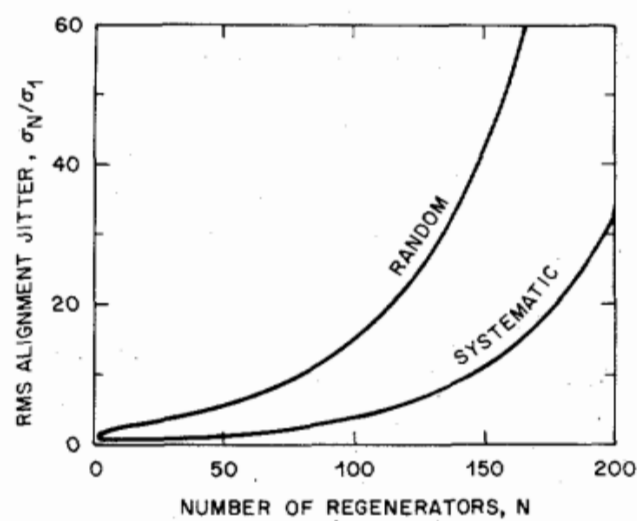

Fig. 5. Illustration of exponential growth of alignment jitter in the last regenerator of a chain when the regenerators have identical jitter peaks of $0.2 \mathrm{~dB}$.

is normalized to $0 \mathrm{~dB}$ at low frequencies for a single regenerator, and the horizontal scale is normalized to the $3-\mathrm{dB}$ point of the jitter filter response in the absence of multiple reflections. The systematic-jitter spectra show typical cancellation effects resulting from a coherent sum of jitter transfer functions: deep minima and a depression of the corner region. In addition, a spectral peak emerges in the region of the jitter peak. The effect of the peak on jitter accumulation can be appreciated by observing that both scales are plotted logarithmically and that the squared rms jitter is proportional to the numerical area under each spectral curve. The random-jitter spectra lack the phase cancellations that tend to pull down the corner in systematic-jitter spectra, so that the jitter peak takes full effect. As a result, integrated random jitter tends to accumulate along a chain more rapidly than integrated systematic jitter in the presence of jitter peaking. This effect may be seen in Fig. 4, which shows the rms timing jitter accumulation at the end of a chain of $N$ regenerators using identical filters. For comparison, we also show the rms jitter accumulation for nonpeaking filters with the same $Q(800)$. These curves follow the familiar fractional power laws [9]-[11], with random jitter the more slowly accumulating. For each curve, the normalization parameter $\sigma_{1}$ is the rms value of the indicated type of jitter emerging from the first regenerator. The peaking results shown here are quite analogous to those found with phase-locked-loop retiming [8], [12] where jitter peaking is usually present to some degree.

In the absence of jitter peaking, it is known that alignment jitter does not accumulate [2], [3], [9], [11]. With peaking present, however, the rms alignment jitter will in fact grow along the chain, as shown in Fig. 5 [2], [3]. The rate of growth is faster for random jitter than for systematic, just as in the case of timing jitter accumulation. Alignment jitter in the last regenerator of a chain must be carefully controlled to avoid excessive decision errors.

In assessing the implications of Figs. 4 and 5, one must keep in mind that peak-to-peak jitter is some characteristic multiple of rms jitter. Multiples of 6-13 have been seen in various systems. If the multiple is 10 , for example, and if $\sigma_{1}$ is $1 / 2^{\circ}$ for random jitter, Fig. 5 yields a peak-to-peak random alignment jitter of about $210^{\circ}$ in the 150th regenerator. Its decision circuit would then be operating with random alignment swings of $\pm 105^{\circ}$ on top of the other static and dynamic misalignments. In most cases, the result would be a very high error rate. Under similar conditions, the full peak-to-peak accumulated jitter implied by the curves of Fig. 4 would have to be accommodated in the elastic store of the receiving terminal.

The above results appear open to question because the use of a transfer function implies a linear relation between the jitter coming into a regenerator and the jitter leaving it. Linearity is readily justified when jitter accumulation is small or when it is concentrated near dc, as in the case of ripple-free filters. However, when jitter accumulates away from $\mathrm{dc}$, as in the case of jitter peaking, the input-output relation may become effectively nonlinear and thereby introduce deviations from the transfer-function predictions. For the small amounts of peaking under consideration here, estimates [3] indicate that nonlinear effects will be quite unimportant.

The next practical question is how to choose an acceptable degree of filter passband ripple. The answer depends strongly on the random variations in a population of manufactured filters. A suitable analytical formalism has been developed to deal with distributions of filter passband shapes and center-frequency mistuning [3]. The shapes include asymmetric as well as symmetric passbands, with or without ripple, as shown in Fig. 6(a). The jitter transfer function [3] corresponding to each case is shown in Fig. 6(b). The connection between the complex filter transfer function and the complex jitter transfer function is discussed in the Appendix where the two functions are shown to be effectively related through simple symmetry operations.

To simulate a population of manufactured filters, a whole family of passband shapes, including all but the first in Fig. 6(a), has been represented analytically through a filter model described in [2]. Passband shapes were varied through a single random variable, the effective phase shift between the triple-transit and first-transit signals. If that phase were stepped incrementally through a change of $360^{\circ}$, the passband shapes would run the gamut from a 

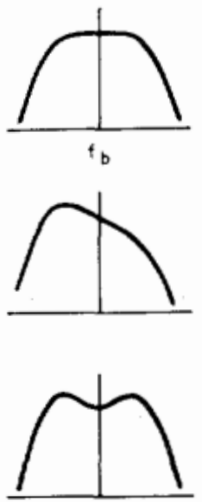

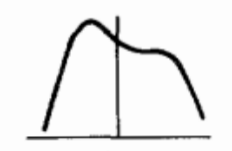

(a)

FILTER

TRANSFER - FUNCTION MODULUS
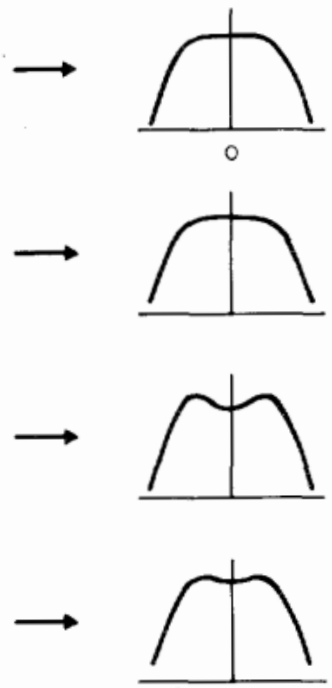

(b)

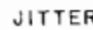

TRANSFER - FUNCTION MODULUS
Fig. 6. (a) Examples of filter passband shapes made possible by shifting the phase of triple-transit interference with the first transit. (b) Corresponding jitter transfer moduli, constructed as described in the Appendix. The jitter modulus narrows and rolls off faster as the baud shifts toward either edge of the passband in (a).

central peak to strong asymmetry to a central dip to reversed asymmetry and back to a central peak. Somewhat more than half the full range, centered on a central dip (largest jitter peak), appears ample for approximate simulation of a prototype filter population for TAT- 8 . With reference to Fig. 1, the interference phase variation was described by a random variation of the transducer separation parameter $n=20+\epsilon$, with $\epsilon$ uniformly distributed between limits $(0.1,0.25)$, equivalent to $(0.1-0.4)$ for symmetry reasons. For purposes of comparison to Fig. 4, where all filters had identical symmetric dips yielding jitter peaks of $0.2 \mathrm{~dB}$, the symmetrically rippled member of the population has been arbitrarily adjusted to a peak of 0.2 $\mathrm{dB}$. The magnitude of individual transducer reflections is thereby determined in the model. The analytical model is too simple to describe flat-topped band shapes, so that ripple effects in the manufactured population are overstated by the model.

The statistical effects on jitter accumulation are illustrated in Fig. 7. The solid lines represent a fully stochastic computation [3] of rms systematic and random jitter accumulation. The surrounding bands extend one standard deviation on either side. In the systematic case, the dashed line corresponds to a chain of identical regenerators whose filter transfer function is an average [3] taken over the filter population. The corresponding curve for random jitter accumulation is too close to its solid curve to be shown.

Fig. 7 contains several striking results. First, the exponential jitter accumulation produced by a chain of peaking regenerators has gone away. The reason is that in the distributed population, the part of the population with jitter peaks is counterbalanced by another part that rolls off rather rapidly. The average jitter transfer function in

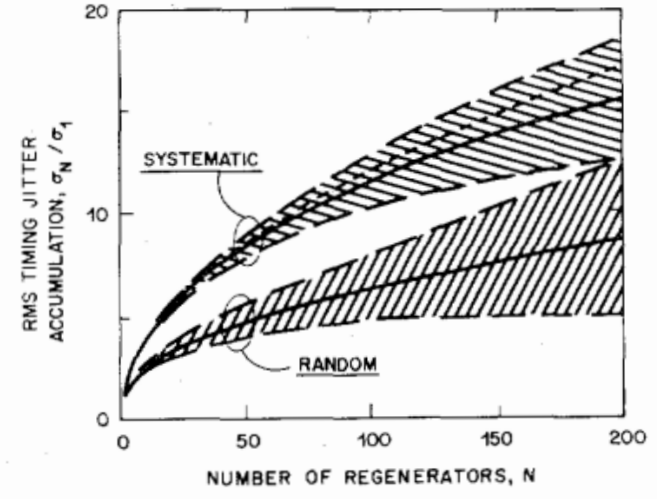

Fig. 7. Timing jitter accumulation along a chain of regenerators whose retiming filters exhibit a distribution of passband shapes. The distribution has been constructed as a "natural" generalization of the single passband shape used to obtain Fig. 4. The solid curves correspond to jitter computed from a fully stochastic computation [3]. Surrounding bands display the regions spanned by \pm 1 standard deviation in each case. For the systematic case, a dashed line shows an approximation described in the text.

the example population is in fact almost maximally flat. Second, the systematic and random accumulation curves have approximately recovered the power-law behavior of nonpeaking regenerator chains, with the systematic jitter accumulating faster than the random jitter. Third, the use of an average filter characteristic in the traditional unvarying-transfer-function computation has yielded a reasonable representation of a chain of regenerators with a distributed filter population.

We conclude that filter passband ripple may be either damaging or innocuous in a long-haul system. The effects of ripple ultimately depend on the magnitude of the associated jitter peaks and on the character and range of variation of passband shapes. Note that the circuits terminating the filters will play an important role since the passband shape of a narrow-band filter is sensitive to the filter terminations.

\section{Transversal Filter "Ringing" Behavior}

A timing-recovery filter must "ring" long enough to maintain a useful timing wave at the decision circuit during the longest string of zeros or NRZ ones expected over system life. Maximum string length was previously estimated to be 62 unit intervals in a 25 -year transoceanic system with a generous safety margin [1]. During the transitionless string, filter deexcitation has to be compensated through the dynamic range of the postfilter amplifier. Filter deexcitation was described [1] by a power-decay factor of $\exp (-2 \pi / Q)$ per unit interval, appropriate to a simple resonator with an inverse fractional bandwidth of $Q$. That decay law led to a mild lower-bound constraint on filter $Q$ implied by the ringing requirement. Since the ringing mechanism in transversal filters is quite different from that in resonator filters, the transversal-filter decay law has been examined, with results described here.

A resonator filter is a minimum-phase infinite-impulseresponse device. It loses a fixed fraction of the trapped 
energy in every transit across the resonant cavity, but the balance remains trapped for later transits. The 3-dB bandwidth is a direct measure of the fractional loss per transit. On the other hand, a transversal (or delay-line) filter (Fig. 1) is a nonminimum-phase finite-impulse-response device with transmission amplitude and phase nearly independent of each other. The filter is essentially a single-pass device that does not ring in the resonator sense. Energy is stored in the filter only during the time needed for the acoustic wave to travel the length of the transmitting and receiving transducer structures. The $3-\mathrm{dB}$ bandwidth is also controlled by propagation: the transmission is ideally a maximum at the synchronous frequency, where the acoustic wavelength matches the period of the transducer finger pattern, and rolls off at other frequencies as the mismatch between wavelength and finger period increases. In evaluating the filter "ringing" behavior, we shall limit consideration to the synchronous case.

Fig. 8 illustrates the coherent buildup of acoustic waves traveling toward the exit face of the transmitter nearest to the receiver. For simplicity, both transducers are assumed to have $N_{T}$ finger periods. We also assume for the moment that the transmitter is excited by a message transition in every unit interval, i.e., that the incoming binary code is $\cdots 101010 \cdots$, so that after the prefilter and squarer of an NRZ timing loop, there is a driving pulse in each time slot. The corresponding transitions are shown by the diagrams [13] in the left-hand column. The voltage applied to the transmitter bus bars generates an acoustic wave train of the same length as the transducer at each instant. Although generation is continuous in time, we show the instantaneously generated wave trains only, at instants separated by multiples of the unit interval. Acoustic waves generated in successive cycles cause a progressive buildup in acoustic amplitude; at the transmitter output face, the amplitude is $N_{T}$ times that produced by a single period of the transducer. Wave sums are indicated at the bottom for $N_{T}=8$. In $\mathrm{CW}$ operation, a fully enhanced wave uniformly spans the entire receiver, whose $N_{T}$ periods provide another multiplication by $N_{T}$ in the received signal. Fig. 8 also illustrates the linear buildup and decay of the acoustic wave when excitation begins and ceases. The linear taper in a transversal filter is analogous to the exponential taper in a resonator filter and is similarly responsible for the ringing. For a random binary message, acoustic generation occurs, on the average, during one out of two cycles, so that on average, the transmitter-wave sum is half as large as that shown. Fluctuations about the average tend to be insignificant for typical retiming filters with hundreds of finger periods in the transducers. Note that the transmitted wave still spans the entire receiver in the presence of continuing traffic, so that there is no additional factor-of- 2 loss of amplitude in the receiver.

The above idealized model has been used to obtain the minimum filter transmission when $m$ consecutive transitionless time slots are imbedded in a long wave train whose mean transition probability outside the $m$ intervals is $1 / 2$. Results are shown in the upper curve in Fig. 9 where

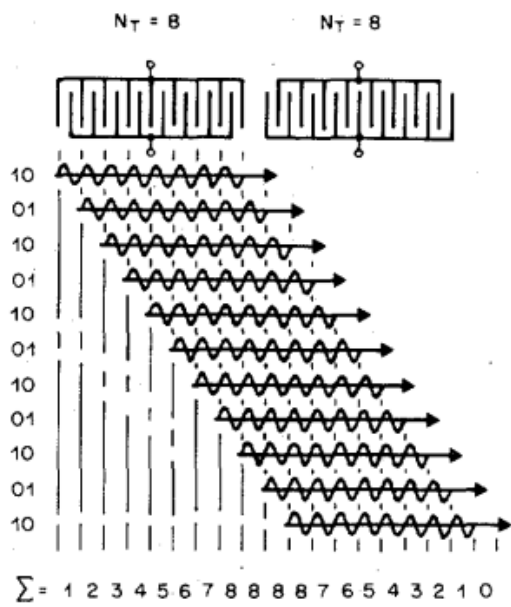

Fig. 8. Illustration of the coherent buildup of a surface acoustic wave by a superposition of wavetrains generated by code transitions in successive unit intervals. Startup and shutdown are characterized by linear signal tapers.

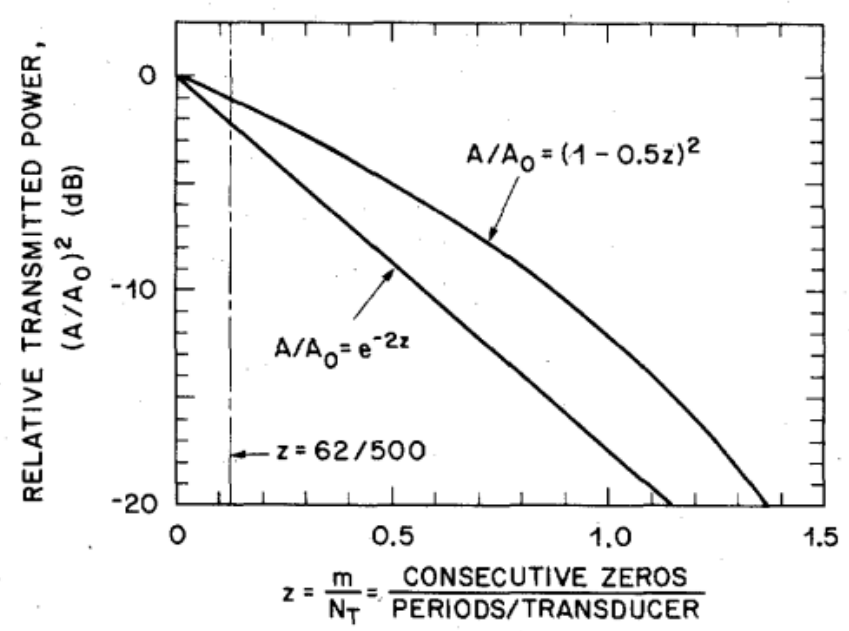

Fig. 9. Minimum transmitted timing-wave power through transversal and simple resonator filters having the same " $Q$ " when normal traffic (transition probability $=1 / 2$ ) is interrupted by a string of $m$ transitionless time slots. The transversal-filter curve is derived from the simplified modeling indicated in Fig. 8.

transmission is normalized to that for $m=0$ and where $m$ is normalized to $N_{T}$ on the abscissa. For comparison, we also show the corresponding transmission (lower curve) for a simple resonator filter having the same $Q$ value as the transversal filter. $Q$ has been approximated here by an expression derived from a familiar model of a SAW transducer [2], [6]; triple-transit effects in the filter have been omitted

$$
Q=\pi N_{T} / 2 .
$$

Since $Q$ scales with $N_{T}$, larger values of the abscissa correspond to lower $Q$ 's.

For a transversal filter on quartz with a $Q$ of $800, N_{T}$ will be roughly 500 . If the message contains one string of 62 zeros or ones, the filter transmission will dip by $1.1 \mathrm{~dB}$, about half as many decibels as the transmission dip of a simple resonator filter of the same $Q$. The factor of 2 in decibels applies for all values of $z$ below roughly 0.2 . As in 
[1], we can relate the minimum transmitted power during a long transitionless string to the dynamic range $D$ of the postfilter amplifier needed to restore the timing wave. With $D$ in decibels, we can combine (1) and the decay law shown in Fig. 9 to obtain

$$
D \simeq-40 \log \left[1-\frac{m \pi}{4 Q}\right] .
$$

With a maximum transitionless string length of 62 time slots during system life, transversal-filter retiming with a $Q$ of 800 should require only $1.1 \mathrm{~dB}$ of dynamic range in the postfilter amplifier.

\section{Thermal Effects in SAW Retiming}

To maintain a low bit-error-ratio (BER) over a specified temperature range (e.g., $0-30^{\circ} \mathrm{C}$ ), the decision cross hair in the eye diagram must be stable against temperature variations. Thermal stability of the eye diagram involves the temperature behavior of three distinct circuit segments: i) the circuit branch that carries the conditioned pulse train to the decision point, ii) the timing-loop electronics, and iii) the timing-recovery filter [1]. For simplicity, we shall start by assuming that part i) is constant over the temperature range of interest. The burden of stability then falls on the complete timing loop, parts ii) and iii). In an NRZ system, part ii) typically includes a prefilter, a squaring circuit, a high-gain limiting postfilter amplifier, and a static phase adjuster. The combination tends to result in a considerable temperature variation of transmission phase, which must be compensated as well as possible [4] through the thermal characteristics of the filter transmission phase.

The thermal behavior of the filter is controlled primarily by the orientation of the quartz substrate [14] and secondarily by the metal-film transducers [15]. Filter transmission phase versus temperature is illustrated in Fig. 10 for a typical high-stability orientation of the quartz. The phase is a parabolic function of the temperature, with the apex, at "turnover temperature" $T$, adjustable from well below $0^{\circ} \mathrm{C}$ to well above $70^{\circ} \mathrm{C}$. The parabola curvature, expressed by the product $c f s_{t}$ (symbols defined in Fig. 10 caption), may depend somewhat on both $T_{t}$ and the metal transducers [15], [16]. Because $T_{t}$ is controllable in manufacture, and because the phase variations of the filter and the electronics are of comparable range, it is possible to achieve good thermal stabilization of the complete timing loop over various temperature ranges of practical interest. This concept was introduced in a terrestrial system design described in [4].

Fig. 11 shows an example more typical of present undersea regenerators employing monolithic integrated circuits. The phase characteristic of the electronics shows a pronounced curvature at the lower temperatures. Since the filter is in cascade with the electronics, phase stabilization only involves a sum of phase shifts. As the figure shows, with the filter turnover temperature in Fig. 10 placed at $60^{\circ} \mathrm{C}$, the total timing-loop phase variation is less than $2^{\circ}$

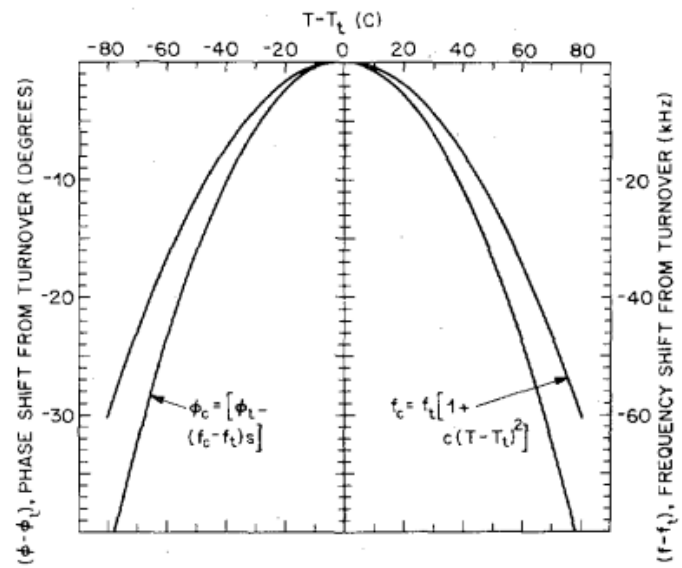

Fig. 10. Dependence of filter center frequency $f_{c}$ on temperature difference from turnover temperature $T-T_{t}$ for a filter with a parabola constant $c=-32 \mathrm{ppb} /\left({ }^{\circ} \mathrm{C}\right)^{2}$. A corresponding phase-temperature parabola is shown for a filter with linear phase slope $s=d \phi / d f=-0.7$ $\mathrm{deg} / \mathrm{kHz} . \phi_{c}=$ transmission phase at $f_{c}, \phi_{t}$ and $f_{t}$ are phase and frequency at the turnover temperature, and the baud frequency $f_{b}$ has been substituted for $f_{t}$ with sufficient accuracy for the figure.

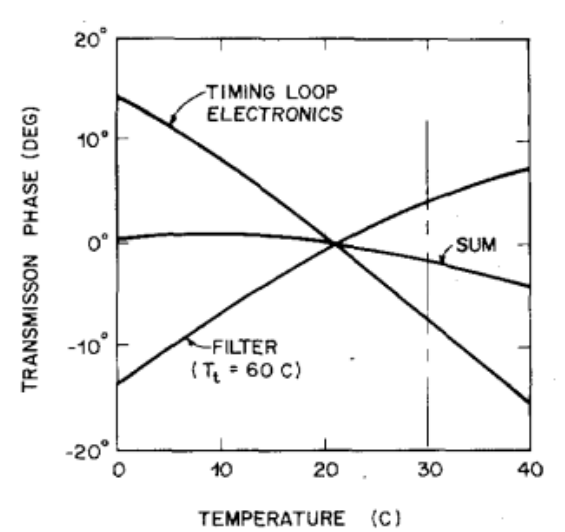

Fig. 11. Example of thermal stabilization of timing-loop electronics by utilizing the SAW filter phase-temperature characteristic. The filter is assumed to behave as in Fig. 10 , with $T_{t}$ placed at $60^{\circ} \mathrm{C}$. The phase origin is arbitrary.

between 0 and $30^{\circ} \mathrm{C}$. Notice that if the circuit carrying the pulse train to the decision circuit, segment i) above, should exhibit significant thermal phase variations, the timing loop could be adjusted for a similar thermal phase variation to maintain the correct decision phase.

The stabilization scheme described above has an unavoidable consequence. For a filter with a linear phasefrequency characteristic (e.g., a narrow-band transversal filter on quartz), one can show that the temperature variation of phase is tracked by a temperature variation of passband center frequency (see Fig. 10). In other words, as temperature moves away from $T_{t}$, the whole passband shifts downward in frequency. For a given operating temperature range $\left(T_{L}, T_{U}\right)$ (lower and upper limits), the frequency shift across the range is minimized by placing $T_{t}$ at $\bar{T}=\left(T_{L}+T_{U}\right) / 2$. However, if $T_{t}$ is shifted away from $\bar{T}$ to stabilize the timing-loop phase, then the frequency shift $\Delta f$ across $\left(T_{L}, T_{U}\right)$ necessarily increases. Since this source of static detuning must be controlled along with other sources, there is a limit to how far $T_{t}$ can be pushed from 


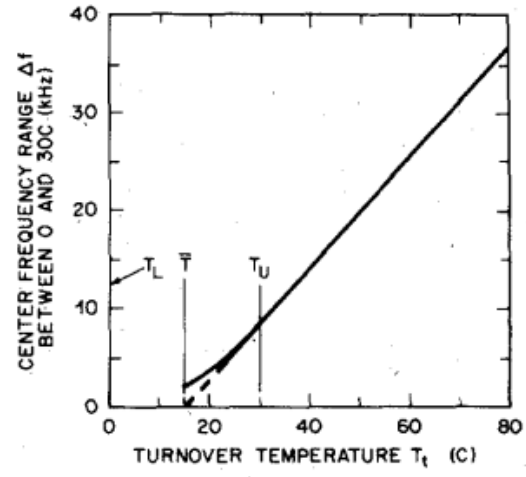

(a)

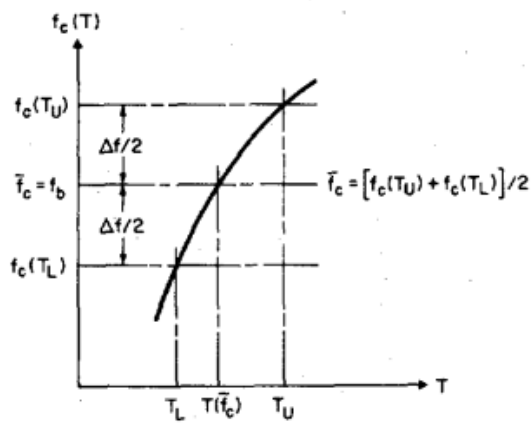

(b)

Fig. 12. (a) Center frequency range swept between 0 and $30^{\circ} \mathrm{C}$ by a filter with parabola constant $c=-32 \mathrm{ppb} /\left({ }^{\circ} \mathrm{C}\right)$, as a function of turnover temperature $T_{t} . f_{c}\left(T_{U}\right)-f_{c}\left(T_{L}\right)$ reverses sign at $T_{t}=\bar{T} \cdot \phi_{c}\left(T_{U}\right)$ $-\phi_{c}\left(T_{L}\right)$ behaves similarly. (b) Example of frequency-trimming target $\bar{f}_{c}=f_{b}$ to minimize thermal frequency deviations from $f_{b}$ between $T_{L}$ and $T_{U}$.

the middle of the temperature range. Fig. 12(a) illustrates how the total frequency range $\Delta f$ across $0-30^{\circ} \mathrm{C}$ depends on $T_{t}$ when the curvature $c$ of the frequency parabola is $-32 \mathrm{ppb} /\left({ }^{\circ} \mathrm{C}\right)^{2}$, a value appropriate to ST-X quartz. For the purpose of this illustration, it is assumed that $c$ is independent of $T_{t}$, a commonly satisfied condition. When $T_{t}>T_{U}$, the curve is a straight line

$$
f_{c}\left(T_{U}\right)-f_{c}\left(T_{L}\right)=-2 c f_{t}\left(T_{U}-T_{L}\right)\left(T_{t}-\bar{T}\right)
$$

where $\bar{T}$ is the center of the operating range. With $T_{t}$ between $T_{U}$ and $\bar{T}$, the curve is a parabola

$$
f_{c}\left(T_{t}\right)-f_{c}\left(T_{L}\right)=-c f_{t}\left(T_{t}-T_{L}\right)^{2} .
$$

When $T_{t}<\bar{T}$, (3) and (4) apply if $T_{L}$ and $T_{U}$ are interchanged.

Fig. 12(b) shows the way to minimize thermal frequency variations when $T_{t} \neq \bar{T}$ : the filter is frequency-trimmed to make the filter center frequency equal to the baud when centered in $\Delta f$. Frequency swings will then be $\pm \Delta f / 2$.

In addition to thermal detuning, other sources of static frequency detuning are trimming errors in filter manufacture, aging of the filter center frequency, and errors (including aging drift) in the terminal master clock. Only the aging and clock frequency offsets have a direct impact on static phase offsets, via the phase slope $d \phi / d f$ of the filter transmission. The phase effects of thermal frequency de-

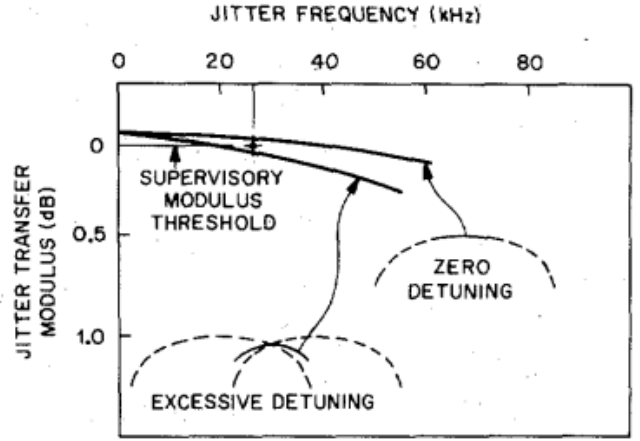

Fig. 13. Illustration of a jitter-transfer-function modulus that meets the mean supervisory requirement at the SL subcarrier frequency (upper curve) and a modulus that falls below the mean supervisory requirement. As shown by sketches, both curves derive from filter passbands of identical shape, with excessive static detuning in the lower case. (See the Appendix.) Curvatures are exaggerated for clarity. The vertical scale is illustrative only.

tuning are compensated as described above, while frequency errors in manufacture translate into phase errors that are corrected by the timing-loop phase adjuster during regenerator assembly.

All frequency detuning mechanisms do have an impact, however, on the supervisory system devised for the TAT- 8 transatlantic cable [17]. Supervisory information is returned to a terminal via the optical channel in the form of tone bursts of an FM subcarrier in the $15-30-\mathrm{kHz}$ range. To maintain a useful subcarrier signal-to-noise $(S / N)$ ratio, the average regenerator must have a jitter transfer function that rolls off very little between the baud and the subcarrier sideband, as shown by the upper curve in Fig. 13. Excessive static frequency detuning of the SAW filters could change the filter transfer function to the lower curve, which rolls off below the threshold of acceptable supervisory $S / N$. The total static frequency detuning must be carefully limited to keep the mean jitter transfer modulus in the acceptable region. The limit tends to tighten as the filter passband shapes become more varied.

\section{SUMMARY}

The jitter effects of retiming-filter passband ripple in long regenerator chains have been examined within the transfer-function approximation. Approximations made along the way are believed to be conservative in the sense that stricter modeling would yield results less harmful to system performance. For a chain of identical regenerators, symmetric ripple has been found capable of causing a) exponential accumulation of timing jitter along the chain, b) random timing jitter accumulation faster than systematic, and c) exponential growth of alignment jitter along the chain, with random jitter again outpacing systematic. However, for a chain of regenerators with a distribution of filter transfer functions, constructed in a realistic manner from the symmetrically rippled case, all the above effects can disappear. When the average filter in the distribution is ripple free, jitter behavior effectively reverts to the ripple-free case. This conclusion is based on ripple not 
in excess of $0.2 \mathrm{~dB}$. For larger ripple, the stochastic variance could become large enough to allow a significant probability of undesirable effects in a randomly assembled chain. Under common manufacturing limitations, it appears necessary to characterize a population of filters to determine whether a system built with them will behave well or badly with respect to jitter.

The ringing behavior of SAW transversal retiming filters has been derived from a highly simplified model. Although the model omits many features of real filters, those features are not expected to alter the ringing behavior appreciably. A SAW transversal filter has been found to drop its output level only half as much in decibels as a simple resonator filter of the same $Q$ after excitation is disrupted by the longest transitionless string expected in 25 years of random traffic. As a result, with random traffic the timing loop can utilize a postfilter amplifier with half the dynamic range previously estimated [1].

Recent developments have been described concerning thermal stabilization of the decision phase in a regenerator. The filter phase-temperature characteristic can be used to stabilize the phase-temperature characteristic of the electronics [4] to within a few degrees in undersea systems. Across the operating temperature range, a penalty in the form of increased thermal shifting of the filter passband has been identified and evaluated. The importance of these shifts will depend on the specific parameters of timing and decision circuits. Implications for center-frequency filter trimming have also been noted.

A simple transformation is described in the Appendix for converting a filter transfer function into the corresponding jitter transfer function. The general features of the latter can be quickly visualized after a little practice.

\section{APPENDIX \\ RElation OF JitTer Transfer Function to Filter TRANSFER FUNCTION}

As shown in [3], the complex jitter transfer function $W(f)$ can be expressed as follows in terms of the complex filter transfer function $H(f)$ : let

$$
H(f)=A(f) e^{j \phi(f)}
$$

Define a low-pass equivalent transfer function by

$$
H_{L}(f)=H\left(f_{b}+f\right) / H\left(f_{b}\right)
$$

where $f_{b}$ is the baud frequency and $f$ is the deviation from the baud, i.e., a baseband frequency. Then the jitter transfer function can be written

$$
W(f)=\left[H_{L}(f)+H_{L}^{*}(-f)\right] / 2
$$

where * means complex conjugate. A similar result, given by Mengali and Pirani [18], was used in [2] to treat the case of detuned resonator filters. We use the symmetries implicit in (7) to obtain a simple construction for $W(f)$.

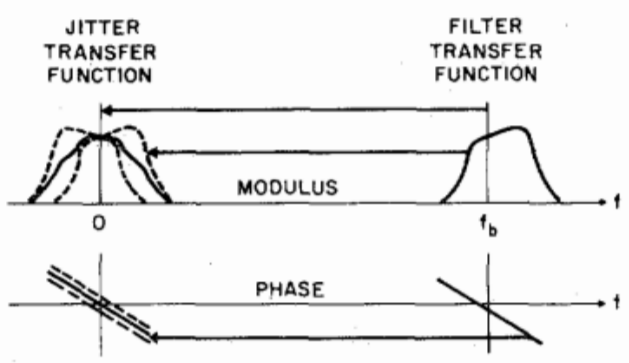

Fig. 14. Transformations showing how a jitter transfer function is constructed from a filter transfer function when the passband phase is sufficiently linear. The jitter transfer function is given by the solid curves on the left.

For that purpose, it is convenient to introduce new amplitude and phase symbols

$$
\begin{aligned}
& B(f)=A\left(f_{b}+f\right) / A\left(f_{b}\right)=B_{e}(f)+B_{o}(f) \\
& \psi(f)=\phi\left(f_{b}+f\right)-\phi\left(f_{b}\right)=\psi_{e}(f)+\psi_{o}(f)
\end{aligned}
$$

where the subscripts " $e$ " and " $o$ " identify the even and odd parts of the functions. Note that $\psi(f)$ is the phase of the filter transfer function measured relative to its phase at the baud. With these relations, we may write

$$
\begin{aligned}
W(f) & =e^{j \psi_{o}}\left(B_{e} \cos \psi_{e}+j B_{o} \sin \psi_{e}\right) \\
& \simeq B_{e} e^{j \psi_{o}}
\end{aligned}
$$

where the last approximation is valid mainly because $\psi$ is effectively linear in frequency across the passband of simple transversal filters, so that $\psi_{e} \simeq 0$.

According to (10), the effective jitter transfer function is formed from the even part of $B$

$$
B_{e}(f)=\frac{A\left(f_{b}+f\right)+A\left(f_{b}-f\right)}{2 A\left(f_{b}\right)}
$$

and the odd part of $\psi$

$$
\psi_{o}=\frac{1}{2}\left[\phi\left(f_{b}+f\right)-\phi\left(f_{b}\right)\right]-\frac{1}{2}\left[\phi\left(f_{b}-f\right)-\phi\left(f_{b}\right)\right]
$$

both defined relative to the baud. These relations have a simple geometrical significance illustrated in Fig. 14: apart from normalization, the jitter transfer function is formed by a) translating the filter transfer function to baseband, with the baud moving to the origin, b) averaging the shifted amplitude response with its mirror reflection in the vertical axis, in accord with (11), and c) averaging the shifted phase response with its transform obtained by inversion through the origin, in accord with (12). Because of construction b), a filter passband with a linear amplitude slope across the baud will have a jitter transfer function that is flat near $f=0$. Construction b) also helps to explain why two-section-Butterworth and simple resonator filters show no jitter peaking with detuning until the detuning exceeds the 3-dB bandwidth [1]. At smaller values of detuning, there is no central dip in the average of the filter transfer function with its reflection through the baud. 


\section{ACKNOWLEDGMENT}

This work has benefited from interactions with many colleagues. Special thanks are due to T. R. Meeker, W. R. Grisé, and W.-S. Tsay; to G. M. Homsey, R. M. Paski, and P. R. Trischitta; and to C. B. Armitage for early information on the feasibility of thermal phase stabilization of the timing loops.

\section{REFERENCES}

[1] R. L. Rosenberg, D. G. Ross, P. R. Trischitta, D. A. Fishman, and C. B. Armitage, "Optical fiber repeatered transmission systems utilizing SAW filters," in 1982 Ultrason. Symp: Proc., IEEE cat. no. 82CH1823-4, pp. 238-246; see also IEEE Trans. Sonics Ultrason., vol. SU-30, pp. 119-126, 1983.

[2] D. A. Fishman, R. L. Rosenberg, and C. Chamzas, "Analysis of jitter-peaking effects in digital long-haul transmission systems using SAW filter retiming," IEEE Trans. Commun., to be published.

[3] C. Chamzas, "Accumulation of jitter: A stochastic model," AT\&T Bell Lab. Tech. J., to be published.

[4] C. B. Armitage, "SAW filter retiming in the AT\&T $432 \mathrm{Mb} / \mathrm{s}$ lightwave regenerator," in Proc. 10th ECOC, Sept. 1984.

[5] R. M. White and R. W. Voltmer, "Direct piezoelectric coupling to surface elastic waves," Appl. Phys. Lett., vol. 7, pp. 314-316, 1965.

[6] W. R. Smith, H. M. Gerard, J. H. Collins, T. M. Reeder, and H. J. Shaw, "Analysis of interdigital surface wave transducers by use of an equivalent circuit model," IEEE Trans. Microwave Theory Tech., vol. MTT-17, pp. 856-864, 1969.

[7] R. L. Rosenberg, "Wave-scattering properties of interdigital SAW transducers," IEEE Trans. Sonics Ultrason., vol. SU-28, pp. 26-41, 1981.

[8] E. Roza, "Analysis of phase locked timing extraction circuits for pulse code modulation," IEEE Trans. Commun., vol. COM-22, pp. $1236-1249,1974$.

[9] C. J. Byrne, B. J. Karafin, and D. B. Robinson, Jr., "Systematic jitter in a chain of digital regenerators," Bell Syst. Tech. J., vol. 42, pp. 2679-2714, 1963.

[10] O. E. de Lange, "The timing of high-speed regenerative repeaters," Bell Syst. Tech. $J$., vol. 37, pp. 1455-1486, 1958.

[11] H. E. Rowe, "Timing in a long chain of regenerative binary repeaters," Bell Syst. Tech. J., vol. 37, pp. 1543-1598, 1958.

[12] J. Wu and E. L. Varma, "Analysis of jitter accumulation in a chain of digital regenerators," in Globecom '82 Conf. Rec., vol. 2, 1982, pp. 653-657.

[13] W. R. Bennett, "Statistics of regenerative digital transmission," Bell Syst. Tech. J., vol. 37, pp. 1501-1542, 1968

[14] J. F. Dias, H. E. Karrer, J. A. Kusters, J. H. Matsinger, and M. B. Schulz, "The temperature coefficient of delay time for X-propagating acoustic surface waves on rotated Y-cuts of alpha quartz," IEEE Trans. Sonics Ultrason., vol. SU-22, pp. 46-60, 1975.

[15] J. Minowa, N. Nakagawa K. Okuno, Y Kobayaski, and M. Morimoto, " $400 \mathrm{MHz}$ SAW timing filter for optical fiber transmission systems," in 1978 Ultrasonics Symp. Proc., IEEE cat. no. 78CH1344-1SU, pp. 490-493.

[16] Y. Shimizu and Y. Yamamoto, "SAW propagation characteristics of complete cut of quartz and new cuts with zero temperature coefficient of delay," in IEEE 1980 Ultrasonics Symp. Proc., IEEE cat. no. $80 \mathrm{CH} 1602-2$; vol. 1 , pp. $420-423$.

[17] C. D. Anderson and D. L. Keller, "The SL supervisory system," pp. 991-997, this issue.

[18] U. Mengali and G. Pirani, "Jitter accumulation in PAM systems," IEEE Trans. Commun., vol. COM-28, pp. 1172-1183, 1980.

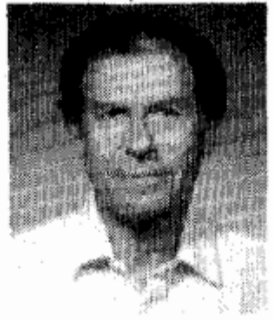

Robert L. Rosenberg (M'73-SM'80) was born in Passaic, NJ, on April 10, 1924. He received the S.B. degree in electronic physics from Harvard University, Cambridge, MA, in 1946, and the M.S. and Ph.D. degrees in physics from Syracuse University, Syracuse, NY in 1952 and 1957, respectively.

$\mathrm{He}$ joined the Research Division of Bell Laboratories in 1955, and has worked in the fields of semiconductors, magnetics, lasers and optics, and ultrasonics. He is coinventor of the "jet-stream" dye laser. He is currently a Distinguished Member of the Technical Staff, Transmission Systems Division, AT\&T Bell Laboratories, Holmdel, NJ, with system and consulting responsibilities in the SAW timing-recovery filter program for undersea and other digital optical-fiber transmission systems.

Dr. Rosenberg was a corecipient of the 1979 Best Paper Award of the Group on Sonics and Ultrasonics, and he serves on the Technical Program Committee of the Ultrasonics Symposium. He is a member of the American Physical Society, Sigma Xi, and Sigma Pi Sigma.

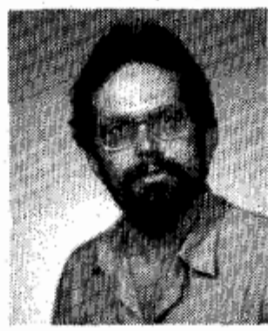

Christodoulos Chamzas (S'75-M'79) was born in Komotini, Greece, in 1951. He received the Diploma degree in electrical and mechanical engineering from the National Technical University of Greece, Athens, Greece, in 1974 and the M.S. and $\mathrm{Ph} . \mathrm{D}$. degrees in electrical engineering from the Polytechnic Institute of New York, Farmingdale, NY.

From 1979 to 1982 he was an Assistant Professor with the Department of Electrical Engineering, Polytechnic Institute of New York, where is is currently an Adjunct Associate Professor. Since 1982 he has been with AT\&T Bell Laboratories, Holmdel, NJ. His primary interests are in signal processing and communication systems. He has held summer positions in Greece, Portugal, and England as well as at Bell Laboratories.

Dr. Chamzas is a member of the Technical Chamber of Greece and Sigma Xi.

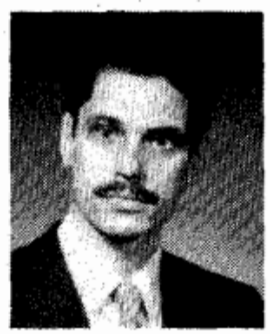

Daniel A. Fishman was born in New York, NY on September 17, 1955. He received the B.S. degree (summa cum laude) in physics from the City College of New York, New York, NY in 1978 and the S.M. degree from the Massachusetts Institute of Technology, Cambridge, in 1981.

$\mathrm{He}$ has worked in the fields of fiber optics, electrodynamics applied to electronic countermeasures, and magnetostatics at various institutions. Since completing his thesis at M.I.T. he has been with Bell Laboratories, Holmdel, where he is a Member of the Technical Staff in the Long Haul Lightwave System Development Department. His current responsibilities include laser transmitter testing and evaluation, microwave circuit design, and jitter simulation and analysis.

Mr. Fishman is a member of Phi Beta Kappa and the American Physical Society, and is an associate member of Sigma Xi. He was the recipient of the C.C.N.Y. 1978 Ward Medal in physics. 